\title{
Zertifizierte Brustzentren - qualitätsgesichert und leitlinienkonform
}

\section{R. Kreienberg, A. Wöckel}

\section{Trend oder Gütesiegel?}

$\nabla$

In dem Zeitraum von 2003 bis Ende 2010 erhöhte sich die Zahl der zertifizierten Brustzentren bundesweit von 8 auf 200. Heute lässt sich der Großteil aller Patientinnen mit einem primären Mammakarzinom in einem zertifizierten Brustzentrum therapieren und erhält dabei eine qualitätsgesicherte Versorgung und eine leitlinienkonforme Therapie: Laut den Angaben der Zertifizierungsstelle ONKOZERT wurden im Jahr 2010 insgesamt 52345 Patientinnen mit primärem Mammakarzinom in diesen Zentren behandelt. Berücksichtigt man den Trend zunehmender Neuerkrankungen (57230 im Jahr 2010), resultiert somit hieraus, dass sich in den Zentren mehr als 91,5\% der Patientinnen therapieren lassen.

Die Verminderung der Sterblichkeit am Mammakarzinom ist heute nicht mehr durch die Einführung einzelner, innovativer Ansätze möglich, sondern nur durch die Qualitätsverbesserung der gesamten Diagnose- und Behandlungskette. Die gesamte Kette kann bekanntermaßen nur so stark sein wie ihr schwächstes Glied. In einem auf Brusterkrankungen spezialisierten Zentrum lässt sich aber gerade die Zusammenarbeit der verschiedenen Fachgebiete so optimieren, dass der gesamte Prozessablauf deutlich verbessert werden kann.

Ein Brustzentrum, zertifiziert nach den Richtlinien der DKG/ DGS, ist verpflichtet, EBM-basierte Leitlinien zu befolgen, bestimmte Struktur- und Prozessqualitäts-Standards einzuhalten und die Ergebnisqualität jährlich offen zu legen.

Die wahrscheinlich wichtigsten Instrumente zur Verbesserung der Diagnostik- und Behandlungsdaten von Brustkrebspatientinnen sind Leitlinien-adaptierte Behandlungsstrategien aller im Brustzentrum zusammengeschlossenen Fachdisziplinen sowie die konsequente Überprüfung der Qualität mittels einer sorgfältigen Datenanalyse der eigenen Leistungen und Kontrollen derselben durch jährliche Audits, verbunden mit einem für alle Beteiligten verbindlichen Qualitätsmanagementsystem.

\section{Die Zukunft der Brustzentren \\ $\nabla$}

Die Zertifizierung von Brustzentren zählt heute sicher zu den wesentlichen Pionierleistungen bei der Etablierung von Organzentren. Andere Fachdisziplinen profitieren hier erheblich von den Erfahrungen, die in der Versorgung von Brustkrebspatientinnen gesammelt werden konnten. Nachdem definierte Qualitätsindikatoren in den Zentren nun bundesweit zunehmend erfüllt und umgesetzt werden, wird der zukünftige Schwerpunkt der Beurteilung auf einer Analyse der Ergebnisqualität liegen müssen. So werden Projekte der Versorgungsqualität an Bedeutung gewinnen, die zukünftig evaluieren werden, welchen Einfluss eine leitliniengerechte und zentrumsspezifische Behandlung auf das Überleben der Patientinnen hat.

\section{Bibliografie}

DOI http://dx.doi.org/10.1055/s-0031-1271512

Senologie 2011; 8: 96

(c) Georg Thieme Verlag KG Stuttgart · New York · ISSN 1611-6453

Korrespondenzadresse

Prof. Dr. med. Rolf Kreienberg

Ärztlicher Direktor der

Universitätsfrauenklinik Ulm

Prittwitzstr. 43

89075 Ulm

rolf.kreienberg@uniklinik-ulm.de

\section{PD Dr. med. Achim Wöckel}

Universitätsfrauenklinik Ulm

Prittwitzstr. 43

89075 Ulm

achim.woeckel@uniklinik-ulm.de 